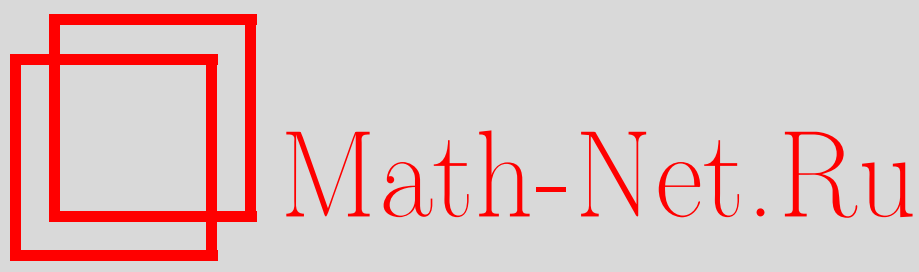

Б. Я. Казарновский, с-вееры и многогранники Ньютона алгебраических многообразий, Изв. РАН. Сер. матем., 2003, том 67, выпуск 3, 23-44

DOI: https://doi.org/10.4213/im434

Использование Общероссийского математического портала Math-Net.Ru подразумевает, что вы прочитали и согласны с пользовательским соглашением

http://www . mathnet.ru/rus/agreement

Параметры загрузки:

IP: 3.85 .73 .92

26 апреля 2023 г., 10:51:54 
УДК $512.7+514.172$

\author{
Б.Я. Казарновский
}

\title{
с-вееры и многогранники Ньютона алгебраических многообразий
}

\author{
К 80-летию Игоря Ростиславовича Шафаревича
}

\begin{abstract}
Алгебраическому подмногообразию комплексного тора сопоставлен евклидов геометрический объект, называемый с-веером. Это сопоставление задает теорию пересечения алгебраических многообразий. с-вееры образуют градуированную коммутативную алгебру с наглядно определяемыми операциями. с-вееры алгебраических многообразий лежат в подкольце рациональных с-вееров. Видимо, другие подкольца могут служить для построения теории пересечений некоторых иных категорий аналитических многообразий. Выявлена связь одной старой проблемы теории выпуклых тел (так называемой проблемы Минковского) с кольцом с-вееров, что позволяет сопоставить любой алгебраической кривой выпуклый многогранник в пространстве характеров тора.

Библиография: 27 наименований.
\end{abstract}

\section{$\S 1$. Введение}

1.1. Кольца рациональных и евклидовых с-вееров. Инварианты алгебраического подмногообразия комплексного тора $(\mathbb{C} \backslash 0)^{n}$ (далее обозначаемого $\left.\mathbb{T}\right)$, заданного как множество совместных нулей невырожденной системы уравнений, можно выразить на языке геометрии многогранников Ньютона [1]-[7]. При переходе к другой невырожденной системе уравнений многообразия изменение многогранников Ньютона практически (кроме случая гиперповерхности) не контролируемо. Инвариантом оказывается произведение многогранников Ньютона системы в определяемом ниже кольце с-вееров (c - начальная буква слова current).

Сопоставим (см. $\S 3) d$-мерному алгебраическому подмногообразию тора $M$ евклидов геометрический объект $\Sigma_{M}$, называемый $d$-мерным с-веером. с-веер это почти (см. определение 1 в $\S 2$ ) $d$-мерный веер рациональных конусов (см. замечание 1 в п. 1.5) с приписанными конусам размерности $d$ целочисленными кратностями. При этом расстановка кратностей должна удовлетворять определенньм линейным условиям. Кратности с-веера $\Sigma_{M}$ положительны, что накладывает сильные ограничения на геометрию веера. с-веер гиперповерхности - это почти (см. п. 1.4) ее многогранник Ньютона. с-вееры образуют коммутативную градуированную $\mathbb{Z}$-алгебру с наглядно определяемыми операциями. Если $M$ задано как множество совместных нулей невырожденной системы с многогранниками Ньютона, то $\Sigma_{M}$ - произведение этих многогранников. Отображение $\Sigma_{M}$ задает (см. $\S 3$ ) теорию пересечений алгебраических многообразий со значениями в кольце с-вееров.

Работа выполнена при поддержке грантов INTAS 00-259, NWO 047.008.005 и РФФИ№ 9901-00245.

(C) Б.Я. КАЗАРНовСКИй, 2003 
Описанные вьше с-вееры будем называть рациональным.ми. Они расположены в пространстве с целочисленной решеткой, их конусы рациональны, а кратности конусов целочисленны.

Возможно построение кольца с-вееров в абстрактном вещественном векторном пространстве $E$ (в контексте многогранников Ньютона это означает переход от полиномов к экспоненциальным суммам с вещественными показателями [15]-[19]). В этом случае кратность конуса $K$ следует определять как нечетную форму объема на $E / E(K)$, где $E(K)$ - порожденное конусом подпространство $E$ (см. $\S 7)$.

При наличии в $E$ фиксированной целочисленной решетки задание нечетной формы объема на $E / E(K)$ эквивалентно (если конус $K$ рационален) заданию кратности конуса $K$, равной интегралу формы по фундаментальному параллелепипеду решетки пространства $E / E(K)$. Абстрактные с-вееры, сосредоточенные на рациональных веерах конусов с заданными целочисленными кратностями, образуют $\mathbb{Z}$-алгебру рациональных с-вееров. Евклидова метрика пространства $E$ также позволяет заменить нечетную форму на $E / E(K)$ числовой (вещественной) кратностью, равной интегралу формы по единичному кубу пространства $E / E(K)$, что упрощает и геометризует описание операций с с-веерами. Такие с-вееры мы называем евклидовыми. Точное определение с-вееров и операций с ними приведены в $\S 2,4-6$. Описание свойств рациональных и евклидовых с-вееров проведем одновременно. Для их различения при необходимости используются выражения "в рациональном" или "в евклидовом случае".

Кольца с-вееров по смыслу и структуре близки к кольцам когомологий торических многообразий [7]. В частности, $\mathbb{Z}$-алгебра рациональных с-вееров была построена в [27] в контексте теории торических многообразий как реализация торического кольца Чжоу, т.е. индуктивного предела колец когомологий неособых торических многообразий (кратности конусов в [27] называются весами Минковского).

Сопоставление $M \rightarrow \Sigma_{M}$ строится непосредственно по идеалу многообразия, а также как асимптотическая плотность многообразия (см. $\S 3$ ). Описание $\Sigma_{M}$ на языке торических многообразий см. в п. 1.5.

В настояшее время известны различные реализации торического кольца Чжоу и его евклидовых аналогов [7], [8], [11] [26], [27]. с-вееры, а также их описание в виде дифференщиальных форм (см. ниже п. 1.3) позволяют получить еще одну реализацию и еще один евклидов аналог торического кольца Чжоу. Некоторые возможные области применений с-вееров описаны в пп. 1.2, 1.3.

1.2. Проблема Минковского и многогранники Ньютона алгебраических многообразий. Кольца когомологий торических многообразий и их евклидовы аналоги оказались естественно связанными с гипотезой П. Макмюллена о числах Дена-Соммервиля простых многогранников и сьграли важную роль в ее доказательстве [10]-[13]. Понятие с-веера выявляет связь этой задачи с еще одной задачей геометрии выпуклых тел. Так называемая “проблема Минковского для промежуточных кривизн" в кусочно линейной постановке [22]-[24] прямо формулируется в виде вопроса о структуре кольца с-вееров (см. $\S 8)$. Приведем здесь утверждение об этом кольце, которое является переформулировкой результатов [20], [21].

Пусть $\mathscr{R}_{+}^{k}-$ множество однородных $(n-k)$-мерных абстрактных с-вееров с положительными кратностями и носителями, не принадлежащими никакому собст- 
венному подпространству $E$ (как объяснено в п. 1.4 и $\S 7, \mathscr{R}_{+}^{1}-$ это с-вееры, отвечаюшие полноразмерным выпуклым многогранникам в пространстве $E^{*}$ ).

УТВеРЖДЕнИЕ 1 . Отображение возведения в $k$-ю степень $d_{k}: \mathscr{R}_{+}^{1} \rightarrow \mathscr{R}_{+}^{k}$ ингективно при $1 \leqslant k \leqslant n-1$ и биективно при $k=1, n-1$.

При $k=n-1$ приведенное утверждение - это теорема Минковского о выпуклых многогранниках (см. § 8). Напомним, теорема Минковского утверждает, что, сопоставляя вьпуклому многограннику в $\mathbb{R}^{n}$ набор векторов, направленных по внешним нормалям граней размерности $n-1$ с длинами, равными плошадям, мы (при $n>1$ ) получаем взаимно однозначное соответствие между множеством полноразмерных выпуклых многогранников, заданных с точностью до сдвига, и множеством невырожденных (т.е. не принадлежаших подпространству) наборов попарно не сонаправленных векторов с нулевой суммой.

Проблема Минковского для промежуточных кривизн (в кусочно линейной постановке) состоит в описании образов отображений $d_{k}$, или, иначе говоря, описании степеней (см. ниже п. 1.4) выпуклых многогранников.

Назовем выпуклый многогранник $\Delta$ многогранником Ньютона $(n-k)$-мерного алгебраического многообразия $M$, если с-веер $\Sigma_{M}$ является $k$-ой степенью многогранника $\Delta$. В контексте алгебраической геометрии проблема Минковского может быть сформулирована следуюшим образом. Какие многообразия имеют многогранник Ньютона и какие многогранники, кроме многогранников с вериинами в челых точках, могут быть многогранниками Ньютона алгебрачческих многообразий? У многогранника Ньютона $(n-k)$-мерного алгебраического многообразия (см. п. 1.4) наклоны всех граней рациональны, а плошади $k$-мерных граней целочисленны, если измерять их при помощи соответствуюших $k$-мерных целочисленных решеток.

Как вытекает из теоремы Минковского (см. § 8), многогранник Ньютона имеется у любой алгебраической кривой, если эта кривая не лежит в сдвинутом подторе меньшей размерности. Многообразие, заданное как невырожденное пересечение $k$ гиперповерхностей с одним и тем же многогранником Ньютона $\Delta$, имеет многогранник Ньютона, совпадающий с $\Delta$.

Кроме геометрической (старой) и алгебро-геометрической версий, в контексте с-вееров возникает также следующая занимающая промежуточное положение арифметическая версия проблемы Минковского. Что можно сказать о выпуклом многограннике, если известно, что его $k$-я степень $(1<k<n)$ является рациональным с-веером? Пример - многогранник с вершинами в целых точках, умноженньй на корень $k$-й степени из целого числа.

1.3. Кольцо, порожденное выпуклыми телами. с-вееры можно рассматривать (см. § 7) как потоки (т.е. функционалы на пространстве гладких финитных дифференциальных форм) в комплексификации пространства $E$. Построение с-веера $\Sigma_{M}$ в виде потока описана в $\S 3$ (теорема 1$)$.

Многограннику сопоставлен поток $d d^{c} H$, где $H$ - его опорная функция, продолженная до функции на $E+\sqrt{-1} E$ тождественно по мнимым частям. Будет показано (теорема 3 ), что рассматриваемые как потоки абстрактные с-вееры образуют кольцо, порожденное конечными линейными комбинациями мономов вида $d d^{c} H_{1} \wedge \cdots \wedge d d^{c} H_{k}$, где $H_{i}$ - непрерывные кусочно линейные функции на пространстве $E$, продолженные до функций на $E+\sqrt{-1} E$ тождественно по мнимым частям. Это кольцо подходит для построения теории пересечений многообразий, 
определяемых нулями конечных систем экспоненщиальных сумм с вещественными показателями [15]-[19].

Мономы с $H_{i}$, принимающими целочисленные значения на целочисленной решетке пространства $E$, порождают $\mathbb{Z}$-алгебру рациональных с-вееров. Это последнее утверждение можно доказать, используя описание кольца когомологий торического многообразия (см. ниже п. 1.5). В $\S 7$ доказано только, что с-вееры принадлежат $\mathbb{Q}$-алгебре, порожденной мономами с $H_{i}$, принимаюшими рациональные значения на целочисленной решетке.

Если в качестве $H_{i}$ рассмотреть опорные функции произвольных вьпуклых тел, продолженные тождественно по мнимым частям, то получим кольцо, порожденное выпуклыми телами в двойственном пространстве $E^{*}$. Возможно, это кольцо подходит для построения аналога теории пересечения многообразий, определяемых как корни систем функций, являющихся преобразованиями Фурье обобщенных функций с компактными носителями. В этом случае роль "многогранника Ньютона обобщенной функции" вьполняет вьпуклая оболочка ее носителя.

Если в качестве $H_{i}$ взять произвольные кусочно линейные функции в комплексном векторном пространстве, то соответствующее кольцо, видимо [17], [18], пригодно для построения теории пересечения многообразий, определяемых как корни систем экспонененциальных сумм с комплексными показателями.

1.4. Примеры. Здесь описаны сопоставление $M \rightarrow \Sigma_{M}$ для многообразий размерности $0,1, n-1, n$, связь между $(n-1)$-мерными с-веерами и выпуклыми многогранниками, а также правило возведения многогранника в степень.

Пример 1 (размерности $0, n$ ). Если $M$ - конечное множество точек с кратностями, то $\Sigma_{M}$ - точка нуль с кратностью, равной количеству точек $M$. Если $M-$ это $m$ экземпляров $\mathbb{T}$, то $\Sigma_{M}$ - все пространство с кратностью $m$.

Пример 2 (кривые). Если $M$ - кривая, то $\Sigma_{M}$ - конечное множество рациональных лучей и точка нуль. По направлениям лучей происходят выходы кривой на бесконечность. Приписанная лучу кратность - количество выходов кривой на бесконечность вдоль соответствующего лучу направления.

Рациональный луч с кратностью $m$ можно рассматривать как умноженный на $m$ минимальный целочисленный вектор на луче. Сумма всех (сопоставленных кривой) векторов нулевая (ср. ограничение на кратности из $\S 2$ ). Таким образом, с-веер кривой - это конечный набор целочисленных векторов с нулевой суммой.

Для плоских кривых эти векторы являются сторонами определенного с точностью до сдвига выпуклого многоугольника (многоугольника Ньютона кривой). Произведение двух с-вееров - точка нуль с кратностью, равной удвоенной смешанной площади соответствующих многоугольников. Отсюда алгоритм возведения такого с-веера в квадрат - это алгоритм вычисления площади выпуклого многоугольника по его сторонам.

ПРИМЕР 3 (гиперповерхности и степени многогранников). По выпуклому многограннику $\Lambda$ с вершинами в целых точках (в евклидовом случае - по любому выпуклому многограннику) строится $(n-1)$-мерный с-веер $\Sigma_{\Lambda}$; с-веер гиперповерхности строится по ее многограннику Ньютона.

Носитель с-веера $\Sigma_{\Lambda}$ - веер, образованный конусами, двойственными граням $\Lambda$ ненулевой размерности. Любой конус старшей размерности такого веера двойствен ребру $\Lambda$. Приписанная этому конусу кратность - число, на единицу меньшее 
количества лежащих на двойственном ребре точек с целочисленными координатами, или (в евклидовом случае) длина ребра. В $\S 2$ доказано, что $\Sigma_{\Lambda}$ с построенными кратностями, действительно, является с-веером (предложение 3 ).

Обозначим через $\Sigma_{\Lambda}^{k}(n-k)$-мерный веер конусов, двойственных граням $\Lambda$ размерности $k$ и выше, с приписанными конусам размерности $n-k$ кратностями, равными умноженным на $k$ ! плошадям (в рациональном случае плошадь грани измеряется при помощи $k$-мерной целочисленной решетки) двойственных им $k$-мерных граней (по определению $\Sigma_{\Lambda}=\Sigma_{\Lambda}^{1}$ и $\Sigma_{\Lambda}^{k}=0$ при $k>n$ ). В $\S 7$ доказано, что $\Sigma_{\Lambda}^{k}$ является $k$-й степенью с-веера $\Sigma_{\Lambda}$ в кольце с-вееров (предложение 12). Ниже мы иногда назьваем с-веер $\Sigma_{\Lambda}^{k} k$-й степенью многогранника $\Lambda$.

Из сказанного вытекает, что $n$-я степень многогранника (т.е. $\Sigma_{\Lambda}^{n}$ ) - точка 0 с кратностью, равной умноженному на $n$ ! объему многогранника. Отсюда следует, что (cр. [3]) произведение $n$ многогранников есть точка нуль с кратностью, равной их смешанному объему, умноженному на $n$ !.

ПРИмеР 4 (с-вееры многогранников и кусочно линейные функции). Пусть $E-$ $n$-мерное векторное пространство с фиксированной целочисленной решеткой или метрикой. Непрерьвная функция в $E$ называется кусочно линейной, если она линейна в каждом из конусов некоторого полного веера конусов. Если целочисленная решетка фиксирована, то предполагается, что функция принимает на ней целые значения.

Если функция $H$ кусочно линейна и $\mathscr{K}$ - соответствующий веер конусов, то при следующем ниже правиле расстановки кратностей на $(n-1)$-остове веера $\mathscr{K}$ возникает структура с-веера. Функция $H$ является опорной функцией выпуклого многогранника, если и только если эти кратности окажутся не отрицательными. Обратно, любой $(n-1)$-мерный с-веер получается таким образом. Функция определяется по с-вееру однозначно с точностью до линейного слагаемого (все эти утверждения доказаны в $\S 7$ ).

Правило расстановки кратностей состоит в следующем. Пусть $K, A_{1}, A_{2}-$ $(n-1)$-мерньй и примыкаюшие $n$-мерные конусы веера $\mathscr{K}$ и $h_{1}, h_{2}$ - точки $E^{*}$, определяюшие функцию $H$ в конусах $A_{1}, A_{2}$. Припишем конусу $K$ кратность, модуль которой равен (целочисленной или евклидовой) длине вектора $h_{2}-h_{1}$. На гиперплоскости конуса $K$ имеются две коориентации. Первая коориентация задается упорядоченной парой полупространств, содержащих $A_{1}, A_{2}$, вторая - вектором $h_{2}-h_{1}$. Припишем кратности знак + , если эти ориентации совпадают, и знак - , если они различны.

\section{5. Связь с торическими многообразиями.}

ЗАмЕчАнИЕ 1. Напомнм, что конечное множество многогранных выпуклых конусов размерностей от нуля до $d$ в вещественном векторном пространстве называется $d$-мерным веером конусов (в пространстве с фиксированной целочисленной решеткой конусы предполагаются рациональными), если:

1) все конусы открыты в порождаемых ими подпространствах;

2) любая грань любого из конусов также является конусом этого веера;

3) пересечение замыканий любых двух конусов веера является их общей гранью.

Конус веера минимальной размерности является подпространством. Веер называется острым, если это подпространство нульмерно. Носитель веера - объединение конусов. Веер называется полным, если его носитель - все пространство. 
Конус $K$ острого рационального веера назьвается простым, если примитивные целочисленные векторы, лежащие на ребрах, образуют базис целочисленной ре-

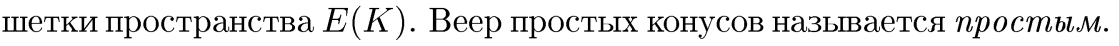

Острому рациональному вееру конусов отвечает торическое многообразие [1], [7]. Многообразие, отвечаюшее полному вееру, компактно, простому - неособо.

Интерпретация алгебро-геометрической части результатов работы на языке торической геометрии состоит в сопоставлении многообразию $M$ торической когомологии, ее выражении в терминах с-вееров и вычислении через многогранники Ньютона.

Замыкание $M$ в неособом торическом многообразии задает цикл, пересечение с которым определяет когомологию. Эти когомологии вместе образуют элемент $\sigma(M)$ индуктивного предела колец когомологий проективной системы неособых торических многообразий, т.е. элемент торического кольца Чжоу [7]. Если $M, N-$ многообразия, заданные как множества совместных нулей двух невырожденных систем $k$ уравнений с одинаковыми наборами многогранников Ньютона [4], то $\sigma(M)=\sigma(N)$. Таким образом, мы получаем симметричную полиаддитивную $k$-форму $\sigma\left(\Delta_{1}, \ldots, \Delta_{k}\right)$ от выпуклых многогранников с вершинами в целых точках, принимаюшую значения в однородной компоненте торического кольца Чжоу. Эта форма равна произведению многогранников в определяемом ниже кольце с-вееров (изоморфном кольцу Чжоу). При $k=n$ функция $\sigma(M)$ числовая, ее значение равно числу точек конечного множества $M$, a $\sigma\left(\Delta_{1}, \ldots, \Delta_{n}\right)$ равно произведению смешанного объема многогранников и $n !$ [4].

c-веер $\Sigma_{M}$ может быть определен на языке торических многообразий следующим образом. Назовем острый веер достаточным для $d$-мерного $M$, если замыкание многообразия $M \subset \mathbb{T}$ в соответствуюшем торическом многообразии компактно. Веер достаточен для $M$, если и только если его носитель содержит определенное в $\S 3$ множество $\Re_{M}$.

Рассмотрим острый веер с носителем $\Re_{M}$ и соответствующее торическое многообразие (как отмечено в $\S 3$, такой веер существует и любой конус такого веера полностью размешается в одной из камер укорочений). Предположим, что этот веер прост (любой веер можно разбить до простого).

Пусть $K-d$-мерный конус веера и $F_{K}$ - замыкание соответствующего конусу $K$ вклеенного $(n-d)$-мерного тора. Сопоставим $K$ кратность, равную индексу пересечения замыкания $M$ с $F_{\mathrm{K}}$. Можно проверить, что кратности конусов, лежаших в одной камере укорочения, одинаковы, и ограничение на кратности, приведенное в $\S 2$, выполняется. Если многообразие $M$ равноразмерно, полученный с-веер совпадает со строящимся в $\S 3$ с-веером $\Sigma_{M}$. Кроме того, $\Sigma_{M}$ может быть получен непосредственной геометрической конструкцией как "асимптотическая плотность" многообразия (теорема 1 в $\S 3$ ).

Связь кольца с-вееров с кольцами когомологий торических многообразий объясняется следуюшим образом. Пусть $\mathscr{K}$ - веер двойственных конусов простого многогранника $\Delta, X$ - соответствуюшее проективное неособое торическое многообразие. Любому $k$-мерному конусу $K \in \mathscr{K}$ отвечает $2(n-k)$-мерный цикл $F_{K}$, определяемьй как замыкание соответствуюшего конусу $K$ вклеенного тора. Таким образом, любому элементу $\sigma \in H^{2(n-k)}(X, \mathbb{Z})$ можно сопоставить набор кратностей $\left\{m(K)=\sigma\left(F_{K}\right)\right\}$, приписанных конусам старшей размерности $k$-мерного остова веера $\mathscr{K}$. Связь кольца с-вееров с когомологиями торических многообразий объясняет следуюший факт (см., например, [27]). Выделяемые та- 
ким способом наборы кратностей - это наборы, удовлетворяющие ограничению на кратности из 2.

Таким образом, при проверке корректности операций и аксиом кольца рациональных с-вееров можно пользоваться когомологиями торических многообразий. Однако при таком не прямом подходе происходят геометрические потери.

Содержание статьи: в $\S 2$ дано определение с-веера; в $\S 3$ приведено построение отображения $M \rightarrow \Sigma_{M}$ и предварительное обоснование теории пересечений; в $\S 4-6$ рассматриваются сложение, проекции и произведение с-вееров; в $\S 7$ дано представление евклидовых с-вееров в виде потоков и некоторые свойства кольца, порожденного выпуклыми телами; в $\S 8$ рассмотрены абстрактные с-вееры и проблема Минковского.

Автор благодарит рецензента за конструктивную критику первого варианта текста.

\section{§ 2. Определение с-веера}

Пусть $\mathscr{K}-d$-мерный веер конусов с приписанными конусам размерности $d$ вещественными числовыми кратностями, расположенный в $n$-мерном вещественном векторном пространстве $E$, в котором фиксирована евклидова метрика или целочисленная решетка. В рациональном случае все рассматриваемые ниже подпространства $E$ предполагаются рациональными (т.е. порожденными векторами целочисленной решетки), а кратности конусов - целочисленньми.

Будем называть $\mathscr{K} d$-мерныц пред-с-веером, если:

1) любой из его конусов является гранью некоторого $d$-мерного конуса (это свойство мы назьваем в дальнейшем условием однородности);

2) кратности конусов удовлетворяют формулируемому ниже ограничению на кратности.

Носителем $d$-мерного пред-с-веера будем называть замыкание объединения $d$-мерных конусов, снабженных ненулевыми кратностями.

Для формулировки ограничения на кратности определим функцию $\xi(A, B)$ на парах подпространств $E$ как коэффициент искажения площади пространства $A /(A \cap B)$ при проекции $\pi: E /(A \cap B) \rightarrow E / B$. Это означает, что для любой области $U \subset A /(A \cap B)$ площадь $\pi(U)$ равна произведению площади $U$ на $\xi(A, B)$ (плошади в факторпространствах измеряются при помоши факторметрик или факторрешеток). Если $A \cup B$ принадлежит собственному подпространству $E$, то $\xi(A, B)=0$ (в этом случае $\operatorname{dim} A /(A \cap B)<\operatorname{dim} E / B)$.

Перечислим (без доказательства) несколько простых свойств функции $\xi$.

Лemma 1. 1) $\xi(A, B)=\xi(B, A)$.

2) Eсли $\operatorname{dim} A+\operatorname{dim} B=\operatorname{dim} E$, то $\xi(A, B)$ равно обвему параллелепипеда, составленного из фундаментальных параллелепипедов решеток или (в евклидовом случае) ортов әтих подпространств.

3) Функиия $\Xi(A, B, C)=\xi(A, B) \xi(A \cap B, C)$ не меняется при перестановках аргументов.

Продолжим функцию на сдвиги подпространств $\xi(a+A, b+B)=\xi(A, B)$ и определим для $d$-мерного веера конусов $\mathscr{K}$ с приписанными конусам размерности $d$ кратностями $m(K)$ функцию на множестве $(n-d)$-мерных афффинных подпро- 
странств $E$ как

$$
\xi_{\mathscr{K}}(A)=\sum \xi(A, E(K)) m(K),
$$

где суммирование идет по конусам веера старшей размерности, пересекающимся с $A$, а через $E(K)$ обозначено порожденное конусом $K d$-мерное подпространство $E$.

ОГРАНИЧЕНИЯ НА КРАТНОСТИ. Для любого $(n-d)$-мерного подпространства $A$ функиия $\xi_{\mathscr{K}}$ постоянна на множестве всех параллельных $А$ аффинных подпространств, не пересекающих $(d-1)$-мерного остова веера $\mathscr{K}$.

Два $d$-мерных пред-с-веера с одним и тем же носителем назовем әквивалентны$\mathcal{м} u$, если любым двум имеюшим $d$-мерное пересечение конусам приписаны одинаковые кратности.

ОПРЕДЕЛЕНИЕ 1. $d$-мерным с-веером (Или однородным с-веером степени $n-d)$ называется класс эквивалентности $d$-мерных пред-с-вееров; с-веер - формальная сумма однородных с-вееров разных степеней.

ЗАмечАниЕ 2 . Пусть $\mathbb{T}_{1}, \mathbb{T}_{2}$ - подторы $\mathbb{T}_{0}$. В общем случае $\mathbb{T}_{1} \cap \mathbb{T}_{2}$ является объединением конечного числа сдвигов некоторого подтора коразмерности, равной $\operatorname{codim} \mathbb{T}_{1}+\operatorname{codim} \mathbb{T}_{2}$. Причина появления функции $\xi$ в том, что количество этих сдвигов равно $\xi\left(\tau_{1}, \tau_{2}\right)$, где $\tau_{i}$ - мнимое подпространство алгебры Ли тора $\mathbb{T}_{i}$, а объемлющее пространство $E$ с фиксированной целочисленной решеткой совпадает c $\tau_{0}$.

ЗАМЕЧАНИЕ 3. Ограничения на кратности тесно связаны с условиями Минковского для выпуклых многогранников (см. далее).

ЗАмЕчАнИЕ 4. Именно ограничения на кратности позволяют дать корректные определения таких операций с с-веерами, как проекции и умножение (см. $\S 5,6)$. Сами по себе они являются условиями корректности определяемого в $\S 6$ умножения с-веера на подпространство (рассматриваемое как с-веер) дополнительной размерности.

ЗАмЕЧАнИЕ 5. Ассоциативность умножения с-вееров основана на приведенном в утверждении 3) леммы 1 “тождестве ассоциативности” для функции $\xi$, которое само по себе является утверждением об ассоциативности умножения рассматриваемых как с-вееры подпространств (следствие 2).

ПРЕДЛОЖЕНИЕ 1. Пусть $v_{1}, \ldots, v_{N}$ - примитивные целочисленные векторы или векторы единичной длины (в евклидовом случае) на лучах некоторого одномерного веера конусов с кратностями $m_{1}, \ldots, m_{N}$. Тогда ограничение на кратности эквивалентно равенству $m_{1} v_{1}+\cdots+m_{N} v_{N}=0$, m.е. одномернье с-вееры с положстельными кратностями - конечные наборы попарно не сонаправленных векторов с нулевой суммой.

ДокАЗАтЕльство. Действительно, если $A$ - нулевое подпространство функционала $\varphi \in E^{*}$, то легко проверить, что ограничение на кратности для сдвигов $A$ эквивалентно равенству $m_{1} \varphi\left(v_{1}\right)+\cdots+m_{N} \varphi\left(v_{N}\right)=0$.

Проверка ограничений на кратности сводится к проверке таких ограничений для одномерных вееров следующим способом. Пусть $\varphi$ принадлежит $(d-1)$-мерному конусу $d$-мерного веера $\mathscr{K}$ с кратностями $\left\{m_{i}\right\}$ Локализация $\mathscr{K}^{\varphi}$ (см. далее 
замечание 6) является веером типа $d$-мерная книга, т.е. состоит из $d$-мерных полупространств $K_{1}, \ldots, K_{N}$ с кратностями $m_{1}, \ldots, m_{N}$, пересекающихся по общему $(d-1)$-мерному подпространству $H$. Ограничения на кратности для такого веера эквивалентны ограничениям на кратности для его веера-факторизации (см. ниже замечание 6), т.е. для одномерного веера.

ПРЕДЛОЖЕНИЕ 2. Ограничения на кратности для $d$-мерного веера $\mathscr{K}$ выполнены тогда и только тогда, когда они выполнены для всех имеющих тип $d$-мерная книга локализаций веера $\mathscr{K}$, или, что то же самое, для всех одномерных вееров, полученных как факторизачии этих локализаций.

ДокАЗАТЕльство. Для доказательства достаточно заметить, что:

1) множество аффинных подпространств с фиксированной $(n-d)$-мерной базой, не пересекаюшихся с $(d-2)$-мерным остовом $d$-мерного веера, связно;

2) при прохождении подпространством $(d-1)$-мерного конуса сохранение значения фунщии $\xi_{\mathscr{K}}$ обеспечивается ограничениями на кратности для соответствующей $d$-мерной книги.

Пусть $\Lambda \in E^{*}$ - полноразмерный выпуклый многогранник, $v_{\Delta} \in E$ - вектор единичной внешней нормали и $m_{\Delta}$ - плошадь его $(n-1)$-мерной грани $\Delta . \mathrm{B}$ рациональном случае предполагается, что $(n-1)$-мерные грани $\Lambda$ лежат на сдвигах рациональных подпространств. Площади $k$-мерных граней такого многогранника можно измерять при помоши целочисленных решеток в порожденных гранями рациональных подпространствах, а вектором нормали грани $\Delta$ назовем тот примитивный вектор целочисленной решетки в $E$, который (как функционал, ограниченньй на многогранник) достигает максимума во всех точках грани одновременно. Легко проверяемое равенство $\Sigma_{\Delta} m_{\Delta} v_{\Delta}=0$ назовем условием Минковского для многогранника $\Lambda$.

Проверим ограничения на кратности для (определенного в п. 1.4) с-веера $\Sigma_{\Lambda}^{k}$.

ПРЕДЛОЖЕНИЕ $3 . \Sigma_{\Lambda}^{k}$ является с-веером.

Действительно, из предложения 2 вытекает, что ограничения на кратности для $\Sigma_{\Lambda}^{k}$ представляют собой совокупность условий Минковского для $(k+1)$-мерных граней многогранника $\Lambda$, рассматриваемых как полноразмерные выпуклые многогранники в порождаемых ими подпространствах.

ЗАмЕчАнИЕ 6. Напомним о двух простых связанных с веерами и переносимых на с-вееры конструкциях. Если вектор $\varphi$ принадлежит замыканию снабженного кратностью $m$ конуса $K$, то снабдим конус $K^{\varphi}=\{k+\mu \varphi: k \in K, \mu \in \mathbb{R}\}$ той же кратностью и назовем его $\varphi$-локализацией $K$. Если $\varphi$ не принадлежит замыканию $K$, то положим $K^{\varphi}=\varnothing$ с кратностью нуль. Для снабженного кратностями веера конусов $\mathscr{K}$ его $\varphi$-локализация $\mathscr{K}^{\varphi}$ - это образуюшее веер множество конусов вида $\left\{K^{\varphi}: K \in \mathscr{K}\right\}$ (этот веер не является острым) с определенными выше кратностями.

Нетрудно убедиться в том, что $\varphi$-локализации эквивалентных пред-с-вееров эквивалентны и ограничение на кратности сохраняется. Таким образом, мы получаем корректно определенную операцию $\varphi$-локализации однородного с-веера.

Еще одна полезная операция - факторизация не острого веера. Если (рациональное в случае рациональных с-вееров) подпространство $E_{1} \subset E$ принадлежит минимальному конусу веера, то образ веера при отображении факторизации 
$\mathrm{E} \rightarrow E / E_{1}$ также является веером конусов. Скажем, что с-веер не острый, если имеется представляющий его не острый пред-с-веер. Легко проверить, что факторизация не острого с-веера определена корректно.

\section{§ 3. с-веер полиномиального идеала}

Проще всего (без учета обоснования) определить сопоставляемый равноразмерному алгебраическому многообразию $M \subset \mathbb{T}$ объект $\Sigma_{M}$ в виде потока на алгебре Ли тора $\mathbb{T}$ (интерпретация с-вееров как потоков содержится в $\S 7$ ). Пусть $\mathscr{M}_{k}-$ поток, заданный как интегрирование по экспоненциальному прообразу алгебраического многообразия, образованного корнями $k$-й степени из принадлежащих $M$ элементов тора.

Teopema 1. Пусть $\operatorname{dim} M=d ; \operatorname{moz} \partial a \lim _{k \rightarrow \infty} \mathscr{M}_{k} / k^{n-d}=\Sigma_{M}$.

Доказательство здесь не приводится. Оно вытекает из теоремы 2 и интерпретации с-вееров как потоков.

Конструкция с-веера, сопоставленного идеалу $I$ кольца полиномов Лорана (т.е. кольца регулярных функций на торе) приведена (без учета кратностей) и обоснована в [14] (анонсирована в [15]). Здесь мы воспроизведем (без обоснования) эту конструкцию с необходимыми пояснениями.

Пусть $A$ - кольцо полиномов Лорана. Обозначим через $E n$-мерное вещественное пространство, сопряженное пространству, содержащему решетку характеров тора $\mathbb{T}$, с двойственной целочисленной решеткой (удобны отождествления $\mathbb{T}=(\mathbb{C} \backslash 0)^{n}$ и $\left.E=\operatorname{Re} \mathbb{C}^{n}\right)$. Множество характеров, входящих в полином Лорана $f$ с ненулевыми коэффициентами, называется носителем полинома. При $\varphi \in E$ определим $\varphi$-степень полинома как максимальное значение $\varphi$ на носителе $f$. Старшая $\varphi$-однородная компонента $f$ называется $\varphi$-укорочением и обозначается через $f_{\varphi}$.

ОПРЕДЕЛЕНИЕ 2. $\varphi$-укорочением идеала $I$ кольца $A$ назьвается идеал $I_{\varphi}$, порожденный полиномами $\left\{f_{\varphi}, f \in I\right\} ; \varphi$-укорочением алгебраического многообразия $M$ нулей идеала $I$ называется многообразие $M_{\varphi}$ нулей идеала-укорочения $I_{\varphi}$.

В определении 2 и всюду далее в этом параграфе предполагается, что соответствие $\{$ идеалы $\} \leftrightarrow\{$ многообразия $\}$ учитывет кратности компонент.

Обозначим через $\Re_{I}$ или $\Re_{M}$ подмножество точек $\varphi \in E$, для которых $I_{\varphi} \neq A$. Зададим в $\Re_{I}$ соотношение эквивалентности $\varphi \sim \psi \Leftrightarrow I_{\varphi}=I_{\psi}$. Связные компоненты классов этой эквивалентности назьваются камерами укорочений идеала (или многообразия).

Множество камер укорочения конечно, а их объединение замкнуто. Каждая камера является открытым коническим подмножеством некоторого рационального подпространства $E$. Максимальная размерность камеры укорочения равна $\operatorname{dim} M$, а соответствуюшее многообразие-укорочение является объединением конечного числа сдвигов подтора размерности $\operatorname{dim} M$, что позволяет приписать камерам максимальной размерности целые положительные кратности.

Существуют $d$-мерные $(d=\operatorname{dim} M)$ вееры конусов с носителем $\Re_{M}$. Такие вееры строятся по некоторым специальным системам образующих идеала $I$, доказательство существования которых - основной технический результат работы [14]. Кроме того, верно, что любая камера укорочения составлена целиком из конусов любого веера с носителем $\Re_{M}[14]$. Поэтому $d$-мерные конусы любого такого веера 
можно снабдить целыми неотрицательными кратностями. Если $M$ равноразмерно, то любая камера укорочения принадлежит замыканию одной из камер максимальной размерности, что гарантирует для таких вееров выполнение условия однородности (см. определение пред-с-веера в начале $\S 2$ ).

ТеОРема 2. Пусть многообразие $M$ равноразмерно и $d$-мерно. Тогда приведенная конструкщия определяет d-мерный с-веер $\Sigma_{M}$ с носителем $\Re_{M}$.

Доказательство части теоремы, не затрагивающей ограничения на кратности, полностью приведено в [14]. Ограничение на кратности является следствием теорем 3 и 4 из [14] и геометрическим отражением того факта, что взятое с кратностями количество точек в слое некоторого разветвленного накрытия над связной базой постоянно. Это накрытие возникает при ограничении на $M$ отображения факторизации $\mathbb{T}$ по $(n-d)$-мерному подтору, алгебра Ли которого порождается фигурируюшим в формулировке ограничений на кратности подпространством $A$ (если $A$ трансверсально всем $d$-мерным камерам, то это отображение собственно и сюръективно).

ЗАмЕчАНИЕ 7. При $\operatorname{dim} M=1, n-1$ камеры укорочений сами являются конусами и образуют веер конусов. Это остается верным для неприводимых двумерных многообразий [14].

ЗАмЕчАниЕ 8. Камеры укорочений не всегда выпуклы. Например, если $M-$ объединение двух 2-мерных подторов 4-мерного тора, то сушествуют три камеры: точка нуль и две 2-мерных плоскости без точки нуль, имеющие кратность 1.

Из работы [14, теорема 2] вытекает

ПРЕДЛОЖЕНИЕ 4. Для любого $\varphi \in \Re_{M} \varphi$-локализачия $\Sigma_{M}^{\varphi}$ с-веера $\Sigma_{M}$ совпадает с с-веером многообразия-укорочения $\Sigma_{M_{\varphi}}$.

Приведем утверждения теории пересечений со значениями в кольце с-вееров:

1) $\Sigma_{M \cup N}=\Sigma_{M}+\Sigma_{N}$;

2) $\Sigma_{M \cap g N}=\Sigma_{M} \Sigma_{N}$ при любом $g \in \mathbb{T} \backslash D(M, N)$, где $D(M, N)$ - некоторое зависящее от $M$ и $N$ собственное алгебраическое подмногообразие тора, пустое для пары многообразий обшего положения (см. предложение 5);

3 ) для любого подтора $\mathbb{T}_{1} \subset \mathbb{T}$ коразмерности, большей или равной $d$, определен согласованный с проекциями алгебраических многообразий гомоморфизм аддитивной группы однородных $d$-мерных с-вееров в аддитивную группу однородных $d$-мерных с-вееров, построенных по тору $\mathbb{T} / \mathbb{T}_{1}$.

Все эти утверждения вытекают из результатов работы [14] и приведенных в § 4-6 определений операций с с-веерами. Коротко объяснение свойств с-веера многообразия-пересечения состоит в следуюшем.

ПРЕДЛОЖЕНИЕ 5. Для любых равноразмерных алгебраических подмногообразий $M, N$ тора существует такое дискриминантное подмногоообразие $D(M, N) \subset \mathbb{T}$, что при $\tau \notin D(M, N)$ с-вееры многообразий $M \cap(\tau N)$ одинаковы. Если камеры укорочений многообразий $M, N$ пересекаются трансверсально, то $D(M, N)=\varnothing$. 
ДоказАТЕЛЬСтво. В случае $\operatorname{dim} M+\operatorname{dim} N \leqslant n$ утверждение очевидно. Пусть $\mathbb{T}_{\varphi}^{M}=\left\{t \in \mathbb{T}: t M_{\varphi}=M_{\varphi}\right\}$, т.е. $\mathbb{T}_{\varphi}^{M}$ - стабилизатор многообразия-укорочения $M_{\varphi}$. Алгебра Ли подтора $\mathbb{T}_{\varphi}^{M}$ - подпространство, порожденное векторами содержащей $\varphi$ камеры укорочения. Многообразие-укорочение $M_{\varphi}$ является прообразом некоторого равноразмерного подмногообразия фактортора $\mathbb{T} / \mathbb{T}_{\varphi}^{M}$ коразмерности, равной коразмерности $M[14]$.

При ненулевом $\varphi \in \Re_{M} \cap \Re_{N}$ размерность подтора $\mathbb{T}_{\varphi}=\mathbb{T}_{\varphi}^{M} \cap \mathbb{T}_{\varphi}^{N}$ равна размерности пересечения содержаших $\varphi$ камер укорочения $M$ и $N$. Обозначим через $M^{\varphi}, N^{\varphi}$ образы многообразий-укорочений при проекции $\mathbb{T} \rightarrow \mathbb{T} / \mathbb{T}_{\varphi}$, и пусть $D_{\varphi}(M, N)$ - прообраз дискриминантного многообразия $D\left(M^{\varphi}, N^{\varphi}\right)$. Из результатов [14] вытекает, что можно положить (используя индукцию по размерности тора) $D(M, N)=\cup_{\varphi} D_{\varphi}(M, N)$, где суммирование идет по конечному множеству ненулевых $\varphi$, выбранных по одному в каждом непустом попарном пересечении камер укорочения многообразий $M, N$.

При большом допустимом (см. § 6) значении $\operatorname{Re} \log \tau$ нетрудно проследить (используя определения $\S 6$ ), что с-веер $\Sigma_{M \cap \tau N}$ равен произведению с-вееров $\Sigma_{M} \Sigma_{N}$.

\section{§ 4. Сложение с-вееров}

Пусть $\mathscr{K}, \mathscr{L}-d$-мерные вееры с превращающими их в пред-с-вееры кратностями $k$ и $l$ соответственно. Объединение их носителей распадается в объединение попарно непересекаюшихся подмножеств трех видов:

$\{K \cap L: K \in \mathscr{K}, L \in \mathscr{L}\}, \quad\{K \backslash \operatorname{supp} \mathscr{L}: K \in \mathscr{K}\}, \quad\{L \backslash \operatorname{supp} \mathscr{K}: L \in \mathscr{L}\}$.

Подмножества первого вида - конусы, второго и третьего - открытые области конусов. Припишем $d$-мерным подмножествам трех указанных видов кратности $k(K)+l(L), k(K)$ и $l(L)$ соответственно.

Пусть $\mathscr{P}$ - веер конусов, носитель которого есть объединение носителей $\mathscr{K}$ и $\mathscr{L}$, такой, что каждое из указанных выше подмножеств является объединением конусов $\mathscr{P}$ (очевидно, что такие вееры сушествуют). Припишем $d$-мерным конусам $\mathscr{P}$ кратности содержащих их подмножеств. Тогда, как нетрудно проверить, мы получим пред-с-веер $\widetilde{\mathscr{P}}$. Если взять другой веер $\mathscr{Q}$ с теми же свойствами, то пред-с-вееры $\widetilde{\mathscr{P}}$ и $\widetilde{\mathscr{Q}}$ эквивалентны. Кроме того, если заменить пред-с-вееры $\mathscr{K}, \mathscr{L}$ на эквивалентные, то класс эквивалентности $\widetilde{\mathscr{P}}$ останется прежним.

Таким образом, мы определили сложение однородных с-вееров одинаковой степени. Ясно, что эта операция ассоциативна и коммутативна, и при переходе к обратному элементу конусы сохраняются, а кратности умножаются на -1 .

Напомним, что сложение с-вееров разных степеней определено формально.

Приведем пример сложения 1-мерных с-вееров на плоскости:

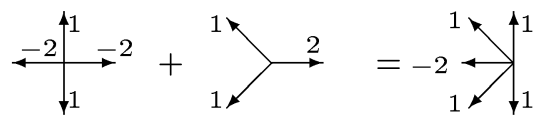

\section{§ 5. Проекции с-вееров}

Пусть $\mathscr{K}-d$-мерный пред-с-веер, $\{m\}$ - набор кратностей его $d$-мерных конусов, $\pi: E \rightarrow E / H$, где $H-$ подпространство $E$ размерности, меньшей или равной $n-d$. Векторы из $\pi(\operatorname{supp} \mathscr{K})$ назовем әквивалентными, если их прообразы пересека- 
ются с одинаковыми наборами конусов веера $\mathscr{K}$. Ясно, что на носителе $\pi(\operatorname{supp} \mathscr{K})$ можно построить веер $\mathscr{K}_{\pi}$, каждый конус которого лежит в своем классе эквивалентности. Покажем, как снабдить $d$-мерные конусы этого веера кратностями $m_{\pi}$.

Пусть $K_{\pi}-d$-мерный конус веера $\mathscr{K}_{\pi}$. Положим $m_{\pi}\left(K_{\pi}\right)=\sum \mu(E(K), H) \times$ $m(K)$, где суммирование идет по конусам $K$ веера $\mathscr{K}$, пересекающимся с $\pi^{-1}\left(K_{\pi}\right)$, а $\mu$ - коэффищиент искажения площади пространства $E(K)$ при отображении $\pi$.

Если $\operatorname{dim} H=n-d$, то $\mu(E(K), H)=\xi(E(K), H)$, и ограничения на кратности $\left\{m_{\pi}\right\}$ (состоящие в том, что все кратности одинаковы) в точности эквивалентны ограничениям на кратности для исходного с-веера $\mathscr{K}$, примененным к сдвигам подпространства $H$. Поэтому мы получаем с-веер степени $d$ в пространстве $E / H$, т.е. конус, совпадающий со всем пространством $E / H$, и приписанную ему кратность. Если кратности исходного с-веера положительны и хотя бы один из его $d$-мерных конусов проектируется без ядра, то эта кратность ненулевая. Если $\operatorname{dim} H<n-d$, то ограничения на кратности $\left\{m_{\pi}\right\}$ вытекает из ограничений на кратности пред-с-веера $\mathscr{K}$, примененных к сдвигам содержащих $H$ подпространств.

Для веера-проекции может не выполняться условие однородности, так как при проекциях алгебраических многообразий свойство равноразмерности не сохраняется. Для его восстановления следует исключить из веера-проекции все конусы, не лежащие в замыкании $d$-мерных конусов.

\section{$\S 6$. Умножение с-веееров}

Операция умножения является переносом на с-вееры операции с веерами конусов, которую мы назовем устойчивым пересечением вееров. Операция устойчивого пересечения вееров, вообще говоря, не является ассоциативной.

Если $\mathscr{A}, \mathscr{B}$ - подмножества векторного пространства $E$, то точку $\mathscr{A} \cap \mathscr{B}$ назовем устойчивой, если ее любая окрестность при достаточно малом $e \in E$ пересекается с $\mathscr{A} \cap(\mathscr{B}+e)$. Множество устойчивых точек пересечения обозначим через $\mathscr{A} \cap \cap^{s} \mathscr{B}$. Например, если $\mathscr{A}, \mathscr{B}$ - подпространства $E$, то $\mathscr{A} \cap^{s} \mathscr{B}$ или совпадает с $\mathscr{A} \cap \mathscr{B}$ (если пересечение трансверсально), или пусто.

Если $\mathscr{K}, \mathscr{N}$ - вееры конусов, то их пересечение $\mathscr{K} \cap \mathscr{N}$ можно определить как веер, образованный конусами $\{K \cap N: K \in \mathscr{K}, N \in \mathscr{N}\}$. Определим веер $\mathscr{K} \cap^{s} \mathscr{N}$, который назовем устойчивым пересечением вееров, следуюшим образом:

1) носитель веера $\mathscr{K} \cap^{s} \mathscr{N}$ - устойчивое пересечение носителей supp $\mathscr{K} \cap^{s}$ $\operatorname{supp} \mathscr{N}$

2) конусы $\mathscr{K} \cap^{s} \mathscr{N}$-множество $\left\{K \wedge^{s} N=K \cap N \cap\left(\operatorname{supp}\left(\mathscr{K} \cap^{s} \mathscr{N}\right)\right): K \in \mathscr{K}\right.$, $N \in \mathscr{N}\}$.

Легко заметить, что множество $K \wedge^{s} N$ или пусто, или совпадает с $K \cap N$, т.е. является конусом веера-пересечения $\mathscr{K} \cap \mathscr{N}$. Устойчивое пересечение замкнутых множеств замкнуто, поэтому объединение конусов вида $K \wedge^{s} N$ замкнуто и, значит, $\mathscr{K} \cap^{s} \mathscr{N}$, действительно, является веером конусов.

В следующей ниже лемме перечислено несколько простых используемых далее свойств устойчивых пересечений вееров.

Лемма 2. 1) Операция устойчивого пересечения вееров перестановочна с локализацией, т.е. если $K \cap N$ содержит ненулевой вектор $\varphi$, то $\mathscr{K}^{\varphi} \cap^{s}$ 
$\mathscr{N}^{\varphi}=\left(\mathscr{K} \cap^{s} \mathscr{N}\right)^{\varphi}, a K \wedge^{s} N=(K \cap N) \cap\left(K^{\varphi} \wedge^{s} N^{\varphi}\right)$.

2) Операчия устойчивого пересечения перестановочна с факторизацией, т.е. если $K, N$ - минимальные конусы вееров $\mathscr{K}, \mathscr{N}$, то $K \wedge^{s} N$ является прообразом минимального конуса устойчивого пересечения профакторизованных по $K \cap N$ вееров при отобрахсении факторизации $E \rightarrow E /(K \cap N)$.

3) Если сумма размерностей вееров равна $n+k$, то размерность их устойчивого пересечения не превосходит $k$.

Пусть $\mathscr{P}$ и $\mathscr{Q}-k$-мерный и $l$-мерный вееры с преврашающими их в пред-с-вееры кратностями $p$ и $q$, а $\widetilde{\mathscr{P}}$ и $\widetilde{\mathscr{Q}}$ - соответствуюшие с-вееры. Назовем точку $e \in E$ допустимой для пары вееров, если множество $D(e)=\operatorname{supp} \mathscr{P} \cap(e+\operatorname{supp} \mathscr{Q})$ состоит только из трансверсальных пересечений конусов.

При $k+l<n$ положим $\widetilde{\mathscr{P}} \widetilde{\mathscr{Q}}=0$.

При $k+l=n$ произведение $\widetilde{\mathscr{P}} \widetilde{\mathscr{Q}}-$ это 0-мерный с-веер, т.е. точка нуль, взятая с целочисленной кратностью $m(0)$.

Кратности при $k+l=n$ задаются следующим образом. Выбрав некоторую допустимую точку $e \in E$, положим

$$
m(0)=\sum_{x \in D(e), x=P \cap(Q+e)} \xi(E(P), E(Q)) p(P) q(Q) .
$$

Напомним, что $E(K)$ - порожденное конусом $K$ подпространство $E$, а функция $\xi$ определена в $\S 2$.

СлЕДСТВИЕ 1. Пусть $E_{1}, E_{2}$ - подпространства Е дополнительной размерности, рассматриваемые как с-вееры. Тогда их произведение - точка нуль с кратностью $\xi\left(E_{1}, E_{2}\right)$.

Приведем пример умножения 1-мерных с-вееров на плоскости:

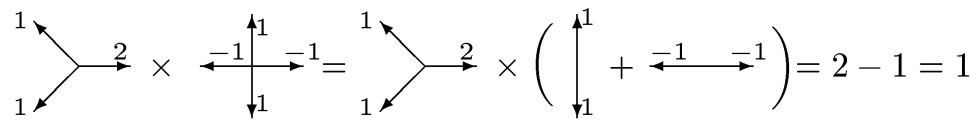

ПРЕДЛОЖЕНИЕ 6 . Кратность $m(0)$ не зависит от произвола в выборе допустимой точки е.

ЗАмЕчАниЕ 9. Этот факт можно понимать как наличие индекса пересечения с-вееров дополнительных размерностей.

ДОКАЗАТЕЛЬСТВО ПРЕДЛОЖЕНИЯ 6 вытекает из следующих соображений.

1. Рассмотрим множество $\mathscr{D}$ всех таких точек $e \in E$, что пересечение supp $\mathscr{P} \cap$ $(e+\operatorname{supp} \mathscr{Q})$, кроме точек пересечения конусов старших размерностей, содержит только точки дискретного пересечения конусов суммарной размерности $n-1$.

2 . При непрерывном изменении $e$ (в области допустимых точек) $m(0)$ не изменяется. Любые две допустимые точки можно соединить лежащей в $\mathscr{D}$ кривой, содержащей только конечное число недопустимых точек.

3. Ограничения на кратности (в локальной форме; см. $\S 2$ ) гарантируют, что изменения $m(0)$ как функции на кривой не происходит также при проходе через не допустимую точку кривой. 
При $k+l=n+d, d>0$, конструкция умножения пред-с-вееров состоит из:

1) правила задания кратностей $d$-мерных конусов веера $\mathscr{P} \cap^{s} \mathscr{Q}$ (это определенное выше устойчивое произведение вееров);

2) проверки ограничения на кратности.

Задание кратностей при $k+l=n+d, d>0$, сводится к случаю $k+l=n$ при помощи локализации и факторизации. Пусть $K-d$-мерный конус веера $\mathscr{P} \cap \cap^{s} \mathscr{Q}$, $\varphi \in K$ и $\mathscr{P} \varphi, \mathscr{Q}^{\varphi}$ - локализации вееров $\mathscr{P}, \mathscr{Q}$. Минимальные конусы этих вееров содержат подпространство $E(K)$. Обозначим через $\mathscr{P}_{f}^{\varphi}, \mathscr{Q}_{f}^{\varphi}$ результат факторизации вееров-локализаций по подпространству $E(K)$. Согласно изложенному в $\S 2$, рассматриваемые как вееры с кратностями, они являются пред-с-веерами в пространстве $E / E(K)$. Поскольку веер $\left(\mathscr{P} \cap^{s} \mathscr{Q}\right)_{f}^{\varphi}$ - точка нуль, то согласно лемме 2 $\mathscr{P}_{f}^{\varphi} \cap^{s} \mathscr{Q}_{f}^{\varphi}=(0)$. Ранее для подобного случая построена кратность $m(0)$. Эту кратность и припишем конусу $K$.

Для проверки ограничений на кратности при $k+l=n+d, d>0$, в локальной форме (предложение 2$)$ или, иначе говоря, в окрестности любого $(d-1)$-мерного конуса $H$ веера $\mathscr{P} \cap^{s} \mathscr{Q}$, можно, выбрав $\varphi \in H$, заменить $\mathscr{P}, \mathscr{Q}$ сначала на их $\varphi$-локализации, а затем на факторизации по пространству $E(H)$. Таким образом, проверка ограничений сведена к случаю $k+l=n+1$, при котором веер $\mathscr{P} \cap^{s} \mathscr{Q}$ одномерен.

В этом случае достаточно (ср. предложение 1) проверить ограничения на кратности для сдвигов гиперплоскости $A$, трансверсальной любому из ненулевых конусов вееров $\mathscr{P}, \mathscr{Q}, \mathscr{P} \cap^{s} \mathscr{Q}$. Пусть $h \in E^{*}$ - уравнение гиперплоскости $A$. Если точка $e$ допустима, то множество $D(e)=\operatorname{supp} \mathscr{P} \cap(e+\operatorname{supp} \mathscr{Q})$ состоит из трансверсальных пересечений конусов, сумма размерностей которых равна $n$ или $n+1$, т.е. $D(e)$ - конечно-звенная ломаная линия. Каждое из звеньев - пересечение конусов суммарной размерности $n+1$. Звену $\lambda=P \cap Q$ припишем кратность $m(\lambda)=\xi(E(P), E(Q))$, где $\xi$ - функция на парах подпространств, определенная в $\S 2$.

ЛЕмма 3. Пусть $\gamma-$ вершина ломаной $D(e)$. Обозначим через $\left\{\alpha_{-}\right\} u\left\{\alpha_{+}\right\}$ множества звеньев ломаной, выходящих из вериинь $\gamma$ в область соответственно меньших и бо́льших значений $h$. Тогда

$$
\sum_{\alpha_{-}} m\left(\alpha_{-}\right) \xi\left(E\left(\alpha_{-}\right), A\right)=\sum_{\alpha_{+}} m\left(\alpha_{+}\right) \xi\left(E\left(\alpha_{+}\right), A\right) .
$$

ДокАЗАТЕЛЬСТво. Пусть $\gamma$ является пересечением конусов $P \in \mathscr{P}, Q \in \mathscr{Q}$ размерностей $k-1, l$, а $\left\{P_{+}\right\}$и $\left\{P_{-}\right\}$- множества $k$-мерных конусов веера $\mathscr{P}$, содержаших звенья ломаной, направленные от $\gamma$ в область соответственно бо́льших и меньших значений $h$.

Используя утверждение 3 ) леммы 1 , можем переписать слагаемые обеих частей в виде $m\left(\alpha_{ \pm}\right) \xi\left(E\left(\alpha_{ \pm}\right), A\right)=\xi(A, E(Q)) \xi\left(A \cap E(Q), E\left(P_{ \pm}\right)\right)$. После сокращения на $\xi(A, E(Q))$ приходим к локальным ограничениям на кратности веера $\mathscr{P}$ (предложение 2)

$$
\sum_{P_{+}} \xi\left(A \cap E(Q), E\left(P_{+}\right)\right)=\sum_{P_{-}} \xi\left(A \cap E(Q), E\left(P_{-}\right)\right)
$$

в окрестности конуса $P$ для подпространства $A \cap E(Q)$. 
Пересечение $D(e)$ с областью достаточно больших положительных значений $h$ состоит из конечного набора лучей $\left\{\kappa_{+}\right\}$(эти лучи - куски звеньев ломаной), параллельных лежащим в полупространстве $h>0$ одномерным конусам веера $\mathscr{P} \cap^{s} \mathscr{Q}$. Положим $m_{+}=\sum_{\kappa_{+}} m\left(\kappa_{+}\right) \xi\left(E\left(\kappa_{+}\right), A\right)$.

Как следует из правила задания кратностей с-веера-произведения, проверяемые ограничения на кратности - это вытекающее из утверждения леммы 3 равенство $m_{+}=m_{-}$, где $m_{-}$определяется аналогично $m_{+}$с использованием области достаточно больших отрицательных значений $h$.

Нетрудно убедиться в том, что, при замене исходных пред-с-вееров на эквивалентные результат остается в своем классе эквивалентности пред-с-вееров.

Скажем несколько слов об ассоциативности операции умножения. Пусть $\mathscr{P}_{0}$, $\mathscr{P}_{1}, \mathscr{P}_{2}-$ с-вееры размерностей $k_{0}, k_{1}, k_{2}$. Так же, как и выше, проверка ограничений на кратности сводится к проверке равенства $\mathscr{P}_{0}\left(\mathscr{P}_{1} \mathscr{P}_{2}\right)=\left(\mathscr{P}_{0} \mathscr{P}_{1}\right) \mathscr{P}_{2}$ в случае $k_{0}+k_{1}+k_{2}=2 n$. Пару точек $e_{1}, e_{2}$ назовем допустимой, если при всех $K_{i} \in \mathscr{P}_{i}$ тройные пересечения конусов $K_{0} \cap\left(e_{1}+K_{1}\right) \cap\left(e_{2}+K_{2}\right)$ трансверсальны. Точке такого тройного пересечения припишем кратность (см. лемму 1 ) $\Xi\left(E\left(K_{0}\right), E\left(K_{1}\right), E\left(K_{2}\right)\right)$ и обозначим сумму всех кратностей через $\mu$. Так же, как при выводе предложения 6 , можно проверить, что $\mu$ не зависит от выбора допустимой пары $e_{1}, e_{2}$.

Проверка того, что тройное произведение вееров, независимо от расстановки скобок, есть точка нуль с кратностью $\mu$, основана на свойстве симметричности функции трех аргументов $\Xi$ (см. лемму 1$)$. Детали этой проверки (близкой к доказательству леммы 3 ) не приводим, так как ассоциативность умножения с-вееров вытекает из содержащейся в $\S 7,8$ интерпретации этого умножения как умножения дифференциальных форм.

Построение кольца с-вееров закончено. Приведем два используемых ниже очевидных свойства этого кольца.

ПРЕДЛОЖЕНИЕ 7. 1) При $\varphi \in E$ отображение $\varphi$-локализации - эндоморфизм кольца с-вееров.

2) Пусть $\mathscr{A}^{\psi}$ - подкольчо кольца с-вееров, представимых пред-с-веерами, минимальный конус которых содержит прямую $\psi$. Тогда отображсение факторизачии задает гомоморфизм кольца $\mathscr{A}^{\psi}$ в кольчо с-вееров пространства $E / \psi$.

\section{§ 7. с-вееры как дифференциальные формы}

Отождествим $n$-мерное евклидово векторное пространство $E$ с пространством $\operatorname{Re} \mathbb{C}^{n}$. Пусть $f, g, \ldots-$ непрерывные кусочно линейные функции на $\mathbb{C}^{n}$, постоянные вдоль пространства мнимых частей $\operatorname{Im} \mathbb{C}^{n}$. Обозначим через $\mathscr{F}$ кольцо дифференциальных форм на $\mathbb{C}^{n}$, порожденное над $\mathbb{R} 2$-формами вида $d d^{c} f$ (напомним, что $d^{c}$ - разность голоморфной и антиголоморфной частей дифференциала).

Корректность определения $\mathscr{F}$ нуждается в обосновании, поскольку $d d^{c} f-$ форма с обобщенныгми коэффициентами (т.е. так назьваемый поток). Известно [25], что $d d^{c} f_{1} \wedge \cdots \wedge d d^{c} f_{k}$ является корректно определенньм потоком, если $f_{1}, \ldots, f_{k}-$ непрерывные плюрисубгармонические (в частности, выпуклые) функции на $\mathbb{C}^{n}$. Это означает, что если последовательность наборов $k$ гладких плюрисубгармонических функций сходится к $\left\{f_{1}, \ldots, f_{k}\right\}$ локально равномерно, то соответствуюшая последовательность дифференциальных форм слабо сходится к не зависяшему 
от выбора последовательности потоку. О предельном потоке известно, что он (как функционал) определен на финитных формах с локально интегрируемыми коэффициентами и принимает неотрищательные значения на положительных формах, т.е. является неотрицательным потоком типа меры. Теперь корректность определения $\mathscr{F}$ вытекает из того, что любая кусочно линейная функция представима как разность двух выпуклых.

Ниже описано, как $k$-мерному с-вееру $\mathscr{K}$ сопоставить однородный поток $W(\mathscr{K})$ бистепени $(k, k)$, т.е. обращающийся в нуль на биоднородных формах, включающих отличное от $n-k$ количество голоморфных или антиголоморфных дифференциалов.

ТЕОРЕМА 3. Отображение $W$ устанавливает изоморфизм кольца евклидовых с-вееров $\mathscr{A}$ и кольца $\mathscr{F}$.

ЗАмЕЧАнИЕ 10. Доказательство теоремы не использует свойства ассоциативности умножения с-вееров. Поэтому эта ассоциативность - следствие теоремы 3.

ЗАмЕчАниЕ 11. Если изменить определение рациональных с-вееров, допуская рациональные, а не только целочисленные значения кратностей, то все используемые ниже аргументы остаются верными и приводят к следующему результату. Отображение $W$ является изоморфизмом этого кольца на кольцо, порожденное над $\mathbb{Q}$ формами вида $d d^{c} H$, где $H$ - опорная функщия выпуклого многогранника с вершинами в точках с рациональными координатами.

Соответствуюший $k$-мерному с-вееру $\mathscr{K}$ поток $W(\mathscr{K})$ определяется следуюшим образом:

$$
W(\mathscr{K})(\varphi)=\sum_{K} \int_{\left(K+\operatorname{Im} \mathbb{C}^{n}\right)} \omega_{K} \wedge \varphi
$$

где $\varphi$ - дифференщиальная форма степени $2 k$ в $\mathbb{C}^{n}$ с компактным носителем (аргумент потока), суммирование идет по $k$-мерным конусам с-веера, а $\omega_{K}$ - определенная ниже нечетная $(n-k)$-форма на пространстве $E(K)+\operatorname{Im} \mathbb{C}^{n}$.

Напомним, что нечетная форма - это внешняя форма в векторном пространстве со знаком, меняющимся при смене ориентации (т.е. нечетная функция на множестве ориентаций). Внешнее произведение нечетной формы на четную - нечетная форма. Нечетная форма объема в векторном пространстве однозначно определяется объемом некоторой фиксированной ограниченной области этого пространства.

Для $m$-мерного конуса $K \in \mathscr{K}$ с приписанной ему кратностью $m_{K}$ построим нечетную форму объема $u_{K}$ на $E / E(K)$, полагая объем единичного куба евклидовой метрики пространства $E / E(K)$ равным $m_{K}$. Для любого конуса $K$ в пространстве $E$ определим отображение $\rho_{K}:\left(E(K)+\operatorname{Im} \mathbb{C}^{n}\right) \rightarrow E / E(K)$ как композицию умножения на $\sqrt{-1}$ и факторизации по подпространству $E(K)+\sqrt{-1} E(K)$. Положим $\omega_{K}=\rho_{K}^{*}\left(u_{K}\right)$, где необходимое для определения прообраза нечетной формы согласование ориентаций пространств $E(K)+\operatorname{Im} \mathbb{C}^{n}$ и $E / E(K)$ устанавливается, исходя из ориентации ядра отображения $\rho_{K}$ как комплексного векторного пространства.

При выводе теоремы 3 нам потребуется переформулировка ограничений на кратности из $\S 2$ в терминах определенных выше форм $u_{K}$. Согласуем ориентации $k$-мерного конуса $K$ и его $(k-1)$-мерной грани $L$ при помощи “вектора внутренней нормали". Такое согласование позволяет рассмотреть нечетную $(n-k)$-форору 
$u_{K}^{L}$ - обратный образ $u_{K}$ при отображении проекции $E / E(L) \rightarrow E / E(K)$. Переформулируем локальные ограничения на кратности (предложение 2 ) следующим образом.

ПрЕДЛОЖЕНИЕ 8. Для любого $(k-1)$-мерного конуса $L, \quad k$-мерного предс-веера $\mathscr{K}$ справедливо равенство $\sum_{K} u_{K}^{L}=0$, где суммирование идет по всем $k$-мерным конусам пред-с-веера, имеющим $L$ своей гранью.

Пусть $\Delta \subset E^{*}$ - выпукльй многогранник, $K_{\Gamma}-$ двойственный конус его грани $\Gamma, u_{\Gamma}$ - нечетная форма на пространстве $E / E\left(K_{\Gamma}\right)$, построенная описанным вьше способом по кратности, равной площади грани $\Gamma$, и $u_{\Gamma}^{\Lambda}$ - прообраз нечетной формы $u_{\Gamma}$ на пространстве $E / E\left(K_{\Lambda}\right)$, где $\Lambda$ - содержащая $Г$ грань на единищу большей размерности. Применяя предложение 8 к определенному в п. 1.4 пред-с-вееру $\Sigma_{\Delta}^{k}$, получаем (это объяснено в конце $\S 2$ ) переформулировку условий Минковского для $(n-k+1)$-мерных граней многогранника $\Delta$.

СлеДСТвИЕ 2. Для любой $(n-k+1)$-мерной грани $\Lambda$ многогранника $\Delta$ справедливо равенство $\sum_{\Gamma} u_{\Gamma}^{\Lambda}=0$, где суммирование идет по $(n-k)$-мерным граням $\Lambda$.

Приведем (без доказательства) еще один, нужный для вывода теоремы 3 , набор тождеств, связываюших нечетные формы $u_{\Gamma}^{\Lambda}$. Эти тождества эквивалентны формуле объема многогранника как суммы площадей его граней, умноженных на длины опущенных на них высот, если применить ее к $(n-k+1)$-мерной грани $\Lambda$ многогранника $\Delta$.

ЛЕмма 4. Пусть $h_{D} \in E^{*}$ - точка, принадлежащая аффинному подпространству, порожденному гранью $D$ многогранника $\Delta$. Тогда для любой $(n-k+1)$-мерной грани $\Lambda$ многогранника $\Delta$ (в обозначениях следствия 2$)$ $u_{\Lambda}=\sum_{\Gamma} h_{\Gamma} \wedge u_{\Gamma}^{\Lambda}$.

Теперь сформулируем утверждения, разбиваюшие утверждение теоремы 3 на части.

ПрЕДЛОЖЕНИЕ 9. Отображение $W$ устанавливает изоморфизм колеи $\mathscr{A}_{0}$ и $\mathscr{F}$, где $\mathscr{A}_{0}$ - подкольцо кольиа $\mathscr{A}$, порожденное с-веерами первой степени.

ПРЕДЛОЖЕНИЕ 10. Кольцо с-вееров порождено әлементами первой степени, т.е. $\mathscr{A}=\mathscr{A}_{0}$.

Поскольку отображение $W$, очевидно, мономорфно и сохраняет операцию сложения, то предложение 9 вытекает из (см. ниже) предложения 11 и описанного в п. 1.4 правила возведения многогранника в степень (его обоснование помещено в следующем ниже предложении 12). Действительно, так как любая кусочно линейная функция является разностью двух выпуклых, то, применяя формулу поляризации, любой элемент $f \in \mathscr{F}$ степени $m$ можно представить в виде $\sum_{\Delta} \pm\left(d d^{c} H_{\Delta}\right)^{m}$, где $H_{\Delta}$ - опорная функция многогранника $\Delta$. Поэтому (см. предложение 11) $f=\sum_{\Delta} \pm W\left(\Sigma_{\Delta}^{m}\right)$. Теперь из предложения 12 (см. ниже) получаем, что $f=\sum_{\Delta} \pm W\left(\Sigma_{\Delta}\right)^{m}$.

ПРЕДЛОЖЕНИЕ 11. Выполнено равенство $\left(d d^{c} H_{\Delta}\right)^{m}=W\left(\Sigma_{\Delta}^{m}\right)$, әде $\Sigma_{\Delta}^{m}-$ определенный в $n .1 .4$ с-веер. 
ДОКАЗАТЕЛЬСТВо. Используя тождество (верное для любой функции $H$ и гладкой финитной функции $\varphi$ )

$$
\left(d d^{c} H\right)^{m}(\varphi)=\left(d d^{c} H\right)^{m-1}\left(H d d^{c} \varphi\right)
$$

и индуктивное предположение $\left(\Sigma^{0}-\right.$ это c-веер, состоящий из одного совпадающего с $E$ конуса с кратностью единица), получаем $\left(d d^{c} H\right)^{m}(\varphi)=W\left(\Sigma_{\Delta}^{m-1}\right)\left(H d d^{c} \varphi\right)$. Поэтому согласно (1)

$$
\left(d d^{c} H\right)^{m}(\varphi)=\sum_{L \in \Sigma_{\Delta}^{m-1}, \operatorname{dim} L=n-m+1} \int_{\left(L+\operatorname{Im} \mathbb{C}^{n}\right)} \omega_{L} \wedge\left(H d d^{c} \varphi\right) .
$$

Представим подьнтегральное выражение в виде сумм двух слагаемых $d\left(H_{L} \omega_{L} \wedge\right.$ $\left.d^{c} \varphi\right)$ и $-\omega_{L} \wedge d H_{L} \wedge d^{c} \varphi$, где $H_{L}-$ любая точка двойственной конусу $L$ грани многогранника $\Delta$, рассматриваемая как вещественная линейная функция на $\mathbb{C}^{n}$ (такая функция совпадает с $H$ на области интегрирования $L+\operatorname{Im} \mathbb{C}^{n}$ ).

ЛЕмма 5. 1) Сумма интегралов первого слагаемого нулевая.

2) Bторое слагаемое при ограничении на области интегрирования $L+$ $\operatorname{Im} \mathbb{C}^{n}$ становится равным $d\left(\omega_{L} \wedge d^{c} H_{L} \wedge \varphi\right)$.

Первое утверждение леммы - следствие приведенных вьше ограничений на кратности, записанных для с-веера $\Sigma_{\Delta}^{m-1}$. Второе - следствие двух фактов:

a) компонента бистепени $(n-m+1, n-m+1)$ формы $d H_{L} \wedge d^{c} \varphi+d^{c} H_{L} \wedge d \varphi$ на принадлежащем ядру формы $\omega_{L}$ подпространстве $E(L)+\sqrt{-1} E(L)$ нулевая;

b) форма $d^{c} H_{L}$ замкнута.

Применяя формулу Стокса к интегралам второго слагаемого, приходим к выражению $\left(d d^{c} H\right)^{m}(\varphi)$ в виде суммы интегралов по областям $L+\operatorname{Im} \mathbb{C}^{n}$, где суммирование идет по $(n-m)$-мерным конусам $L$ веера $\Sigma_{\Delta}^{m-1}$. При этом по области $L+\operatorname{Im} \mathbb{C}^{n}$ интегрируется форма вида $\left(\sum_{K} \omega_{K} \wedge d^{c} H_{K}\right) \wedge \varphi$, где суммирование ведется по $(n-m+1)$-мерным конусам $K$ веера $\Sigma_{\Delta}^{m-1}$, имеющих $L$ своей гранью. Теперь из утверждения леммы 4 (см. также приведенное вьше определение формы $\left.\omega_{L}\right)$ вытекает, что эта форма равна $\omega_{K}$, что и требовалось доказать. Предложение доказано.

ПРЕДЛОЖЕНИЕ 12. $\Sigma_{\Lambda}^{k} \Sigma_{\Lambda}^{p}=\Sigma_{\Lambda}^{k+p}$.

ДокАЗАТЕльСтво. Из приведенного в $\S 6$ правила задания кратностей вытекает, что равенство $\Sigma_{\Delta}^{l} \Sigma_{\Delta}^{m}=\Sigma_{\Delta}^{l+m}$ достаточно проверить в случае $l+m=n$. Пусть $H$ - опорная функция многогранника $\Delta$ и $H_{e}(x)=H(x+e)$. Применяя утверждения предложения 11 и приведенной ниже леммы 6 , получаем

$$
W\left(\Sigma_{\Delta}^{l} \Sigma_{\Delta}^{m}\right)=\lim _{e \rightarrow 0}\left(d d^{c} H\right)^{l} \wedge\left(d d^{c} H_{e}\right)^{m}=\left(d d^{c} H\right)^{n}=W\left(\Sigma_{\Delta}^{n}\right) .
$$

Лемма 6. Пусть $\mathscr{P}, \mathscr{Q}$ - с-вееры дополнительных размерностей. Тогда $W(\mathscr{P} \mathscr{Q})=\lim _{e \rightarrow 0} W(\mathscr{P}) W_{e}(\mathscr{Q})$, где $W_{e}(\mathscr{Q})$ - сдвиг потока $W(\mathscr{Q})$ на допустимую (см. §6) точку е.

Утверждение леммы легко получить из соображений непрерывности или при помощи прямого вычисления. Детали доказательства мы опускаем.

Пусть $\mathscr{K}$ - веер конусов, двойственных граням простого многогранника $\Delta$ (это означает, что все конусы веера $\mathscr{K}$ симплициальны); $\mathscr{B}$ - кольцо всех с-вееров с конусами из $\mathscr{K}$ и $\mathscr{B}^{k}$ - однородные с-вееры $\mathscr{B}$ степени $k$ (или, что то же самое, $(n-k)$-мерные с-вееры). 
Теорема 4. 1) Кольчо $\mathscr{B}$ порождено әлементами первой степени.

2) Умножение с-вееров задает невырожденное спаривание $\mathscr{B}^{k} \oplus \mathscr{B}^{n-k} \rightarrow \mathbb{R}$.

Любой с-веер содержится в каком-нибудь кольце такого вида. Поэтому предложение 10, а с ним теорема 3 вытекают из теоремы 4. Объединим два утверждения теоремы 4 следуюшим образом.

Лемма 7. Пусть $\mathscr{B}_{0}^{k}-\mathscr{B}^{1}$-порожденная часть $\mathscr{B}^{k}$. Тогда при $0 \leqslant k \leqslant n$ спаривание $\mathscr{B}^{k} \oplus \mathscr{B}_{0}^{n-k} \rightarrow \mathbb{R}$ невырожденно.

ДокАЗАТЕЛЬСтво. Предположим, что $\mathscr{P} \in \mathscr{B}^{k}$ принадлежит ядру спаривания, т.е. при любом $\mathscr{Q} \in \mathscr{B}_{0}^{n-k}$ произведение $\mathscr{P} \mathscr{Q}$ нулевое. Пусть $k$ выбрано максимально большим, т.е. при $m>k$ ядро спаривания в $\mathscr{B}^{m}$ нулевое (очевидно, $k<n$ ). Тогда $\mathscr{P} \mathscr{R}=0$ для любого $\mathscr{R} \in \mathscr{B}^{1}$. Пусть $\Lambda-(n-1)$-мерная грань многогранника $\Delta$ с двойственным конусом $\varphi \subset \operatorname{supp} \mathscr{P}$.

При $k<n-1$, применяя локализацию и факторизацию веера (предложение 7 ), сводим утверждение к случаю веера меньшей размерности (веер-факторизация является двойственным веером простого многогранника $\Lambda$ ).

При $k=n-1$ обозначим через $\Gamma$ многогранник, полученный из $\Delta$ мальм параллельным сдвигом грани $\Lambda$. Тогда (так как многогранник $\Delta$ простой) верно, что $\left(\Sigma_{\Delta}-\Sigma_{\Gamma}\right) \in \mathscr{B}^{1}$. Теперь, как нетрудно заметить, из равенства $\left(\Sigma_{\Delta}-\Sigma_{\Gamma}\right) \mathscr{P}=0$ вытекает, что кратность, приписанная лучу $\varphi$ в с-веере $\mathscr{P}$, нулевая.

\section{§ 8. Абстрактные с-вееры и проблема Минковского}

Кольцо потоков $\mathscr{F}$, в отличие от кольца евклидовых с-вееров, определено независимо от выбора метрики в $\operatorname{Re} \mathbb{C}^{n}$. При инвариантном (не зависяшем от метрики) определении с-вееров в вешественном векторном пространстве $E$ следует рассматривать приписанную конусу $K$ кратность как нечетную форму объема $u_{K}$ на $E / E(K)$, где $E(K)$ - порожденное конусом подпространство $E$. Используя определение 1 и принимая утверждение предложения 8 за формулировку ограничения на кратности, получим определение абстрактного с-веера.

Для определения умножения (определение сложения очевидно) абстрактных с-вееров по правилам, приведенным в $\S 6$, достаточно так же, как в евклидовом или рациональном случаях, построить это умножение для рассматриваемых как с-вееры подпространств $E_{1}, E_{2}$ с нечетными формами $u_{1}, u_{2}$ на факторпространствах $E / E_{1}, E / E_{2}$ (в евклидовом и рациональном случаях вместо форм задаются числовые кратности), т.е. нужно построить "произведение" форм $u_{1}, u_{2}$ - нечетную форму объема $u_{1,2}$ на пространстве $E /\left(E_{1} \cap^{s} E_{2}\right)$, где $E_{1} \cap^{s} E_{2}$ - устойчивое пересечение подпространств (см. §6).

Если подпространства $E_{1}, E_{2}$ не трансверсальны, то положим $u_{1,2}=0$. Если подпространства $E_{1}, E_{2}$ трансверсальны, то, выбрав ориентации пространств $E / E_{i}$ и согласованную с этим выбором ориентацию $E /\left(E_{1} \cap E_{2}\right)$ (в трансверсальном случае $\left.E_{1} \cap^{s} E_{2}=E_{1} \cap E_{2}\right)$, положим $u_{1,2}=u_{1}^{*} \wedge u_{2}^{*}$, где $u_{i}^{*}$-прообраз $u_{i}$ при проекции $E /\left(E_{1} \cap E_{2}\right) \rightarrow E / E_{i}$. Форма $u_{1,2}$, действительно, является нечетной, т.е. меняет знак при смене ориентации (заметим, что $u_{1}^{*}$ и $u_{2}^{*}$ не являются нечетными формами).

Введем обозначения $C_{\mathrm{e}}, C_{\mathrm{r}}, C_{\mathrm{a}}$ для колец евклидовых, рациональных и абстрактных с-вееров. Изоморфизм $W$ в $\S 7$ строился как композиция отображений $C_{\mathrm{e}} \rightarrow$ 
$C_{\mathrm{a}} \rightarrow \mathscr{F}$. Поэтому кольца евклидовых и абстрактных с-вееров изоморфны. Сопоставляя рациональному конусу с кратностью $m(K)$ такую нечетную форму объема на пространстве $E / E(K)$, что объем фундаментального параллелипипеда целочисленной решетки равен $m(K)$, получаем вложение $C_{\mathrm{r}} \rightarrow C_{\mathrm{a}}$.

Сопоставление многограннику $\Delta \subset E^{*}$ c-веера $\Sigma_{\Delta}^{k}$ не зависит от выбора метрики или целочисленной решетки. Абстрактный с-веер $\Sigma_{\Delta}^{k}$ строится по выпуклому многограннику $\Delta$ следуюшим образом.

Ограниченной области $A$ векторного пространства $H$ можно сопоставить нечетную форму объема $\psi_{A}$ в сопряженном пространстве $H^{*}$ такую, что для любой

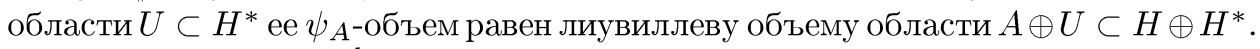
Абстрактный с-веер $\Sigma_{\Delta}^{k}-(n-k)$-мерный остов веера двойственных конусов с нечетными формами объема $\psi_{\Lambda}$, заданными на пространствах $E / E\left(K_{\Lambda}\right)$, где $K_{\Lambda}-$ конус, двойственный $k$-мерной грани $\Lambda$. Таким образом, результаты о возведении многогранника в степень (в частности, теорему Минковского) можно формулировать без использования евклидовой метрики. Нетрудно проверить, что сформулированное в п. 1.2 утверждение о степенях многогранников действительно является переформулировкой результатов [20], [21].

Набор вида $\left\{v_{i}, m_{i}\right\}$, где $v_{i}$ - примитивные целочисленные векторы в пространстве с решеткой $E$, а $m_{i}$ - целые положительные числа такие, что $m_{1} v_{1}+\cdots$ $\cdots+m_{N} v_{N}=0$, назовем данными Минковского. Тогда из теоремы Минковского о выпуклых многогранниках вытекает, что существует и единствен с точностью до сдвига выпуклый многогранник $\Delta \subset E^{*}$ такой, что:

(i) количество $(n-1)$-мерных граней $\Delta$ равно $N$;

(ii) эти грани лежат на сдвигах рациональных подпространств;

(iii) $v_{i}$ - их единичные внешние нормали;

(iv) $m_{i}-$ их плошади.

(Понятие внешней нормали и площади грани многогранника в пространстве с целочисленной решеткой определено в конще § 2.)

с-веер алгебраической кривой (см. п. 1.4) представляет собой данные Минковского. Таким образом, любой кривой может быть сопоставлен выпуклый многогранник.

Во всех известных автору примерах трехмерных многогранников с целочисленными данными Минковского длины их ребер, измеренные с помошью целочисленной решетки, являются суммами квадратичных иррациональностей.

\section{Список литературы}

1. Kemph G., Knudsen F., Mamford D., Saint-Donat B. Toroidal embeddings. 1 // Lect. Notes Math. № 339. N. Y.: Springer-Verlag, 1973.

2. Арнольд В. И., Варченко А. Н., Гусейн-Заде С. М. Особенности дифференцируемых отображений. Монодромия и особенности интегралов. М.: Наука, 1994.

3. Бернштейн Д. Н. Число корней системы уравнений // Функц. анализ и его прилож. 1975. T. 9. №3. C. 1-4.

4. Бернштейн Д. Н., Кушниренко А. Г., Хованский А. Г. Многогранники Ньютона // УМH. 1976. Т. 31. №3. С. 201.

5. Хованский А. Г. Многогранники Ньютона и торические многообразия // Функц. анализ и его прилож. 1977. Т. 11. № 4. С. 56-64.

6. Хованский А. Г. Многогранники Ньютона и род полных пересечений // Функц. анализ и его прилож. 1978. Т. 12. №1. С. 51-61.

7. Данилов В. И. Геометрия торических многообразий // УМН. 1978. Т. 33. №2. C. $85-134$. 
8. Пухликов A. В., Хованский $A$. Г. Конечно аддитивные меры виртуальных многогранников // Алгебра и анализ. 1992. Т. 4. № 2. С. 337-356.

9. Пухликов А. В., Хованский А. Г. Теорема Римана-Роха для интегралов и сумм квазиполиномов по виртуальным многогранникам // Алгебра и анализ. 1992. Т. 4. № 4. C. $188-216$.

10. Stanley R. P. The number of faces of a simplicial convex polytope // Adv. Math. 1989. V. 35. P. 236-238.

11. McMullen P. The polytope algebra // Adv. Math. 1989. V. 78. P. 76-130.

12. McMullen P. On simple polytopes // Invent. Math. 1990. V. 113. P. 419-444.

13. Тиморин B. A. Аналог соотношений Ходжа-Римана для простых выпуклых многогранников // УМН. 1999. Т. 63. №3. С. 113-162.

14. Казарновский Б. Я. Укорочения систем уравнений, идеалов и многообразий // Изв. РАН. Сер. матем. 1999. Т. 63. № 3. С. 119-132; Письмо в редакцию // Изв. РАН. Сер. матем. 2000. Т. 64. № 1. С. 224.

15. Казарновский Б. Я. Экспоненциальные аналитические множества // Функц. анализ и его прилож. 1997. Т. 31. № 2. С. 15-26.

16. Гельфонд O. A. О среднем числе корней систем голоморфных почти периодических уравнений // УМН. 1984. Т. 39. № 1. С. 123-124.

17. Казарновский Б. Я. О нулях экспоненциальных сумм // ДАН СССР. 1981. Т. 257. № 4. C. $804-808$.

18. Казарновский Б. Я. Многогранники Ньютона и корни систем экспоненциальных сумм // Функц. анализ и его прилож. 1984. Т. 18. № 4. С. 40-49.

19. Хованский А. Г. Малочлены. М.: Фазис, 1997.

20. Minkovski H. Volumen und Oberflashe // Math. Ann. 1903. V. 57.

21. Александров А. Д. К теории смешанных объемов выпуклых тел. 1 // Матем. сб. 1937. Т. 2. № 5. С. 947-972; 2 // Матем. сб. 1937. Т. 2. №6. С. 1205-1238; 3 // Матем. сб. 1938. Т. 3. №1. С. 27-46; 4 // Матем. сб. 1938. Т. 3. № 2. С. 227-251.

22. Исследования по метрической теории поверхностей (сборник переводов). М.: Мир, 1980.

23. Погорелов А. В. Многомерная проблема Минковского. М.: Наука, 1975.

24. Бураго Ю. Д. Геометрия поверхностей в евклидовых пространствах // Современные проблемы математики. Фундаментальные направления. Т. 48. М.: ВИНИТИ, 1989.

25. Bedford E., Taylor B. A. The Dirichlet problem for a complex Monge-Ampere equations // Invent. Math. 1976. V. 37. № 2. P. 1-44.

26. Brion $M$. Piecewise polynomial functions, convex polytopes and enumerative geometry // Banach center publ. V. 36. Warszawa: Inst. of Math. Polish Acad. Sci., 1996. P. 25-44.

27. Fulton W., Sturmfels B. Intersection theory on toric varieties. http://arxiv.org/abs/math/ 9403002 .

НТЦ "Информрегистр"

Поступило в редакцию

E-mail: kazarn@borisk.mccme.ru

15.VI.2001 